\title{
Analysis of Niobium precipitates effect on the thermo-mechanical behavior of a NiTiNb Shape Memory Alloy and Modeling
}

\author{
Boris Piotrowski ${ }^{1,2,3, a}$, Tarak Ben Zineb ${ }^{1}$, Etienne Patoor ${ }^{2}$, and André Eberhardt ${ }^{2}$ \\ 1 Laboratoire d'Énergétique et de Mécanique Théorique et Appliquée, Nancy University, CNRS, 2 rue \\ Jean Lamour; 54500 Vandoeuvre-les-Nancy, France \\ 2 Laboratoire de Physique et Mécanique des Matériaux, Paul Verlaine University, ENSAM, ENIM, \\ CNRS, Ile du Saulcy; 57045 Metz cedex 01, France \\ 3 Études et Productions Schlumberger, 1 rue Henri Becquerel, 92140, Clamart France
}

\begin{abstract}
Résumé Commercial $N i_{47} T i_{44} N b_{9}$ Shape Memory Alloy (SMA) has attracted important attention for connection applications thanks to its wide transformation hysteresis. Indeed, it has been shown that reverse transformation temperature (As) can increase by $80^{\circ} \mathrm{C}$, with martensitic reorientation under tensile loading. The aim is to model this behavior in order to design industrial applications, by taking into account the Niobium inclusions effects upon the amplification of this phenomenon for NiTiNb. Niobium precipitates have been identified by Scanning Electron Microscopy (SEM) and X-Ray Diffraction (XRD), and characterized. It seems that the smallest $\mathrm{B}-\mathrm{Nb}$ Niobium inclusions have the most important impact on the phenomenon, explained by Niobium low yield stress and important scattering and number of inclusions. A two phases thermo-mechanical model has been developed. It describes the global effective behavior of an elastic-plastic inclusion (Niobium precipitates) embedded with SMA Matrix. The constitutive law of the matrix is that developed by Peultier et al. and improved by Duval et al.. The elastic-plastic constitutive law for inclusion is a classical one proposed by Simo and Hughes, the Mori-Tanaka scale transition is adopted in order to lead to the effective constitutive law. Numerical results are discussed and compared to experimental ones.
\end{abstract}

\section{Introduction}

The NiTi Shape Memory Effect is often used in industrial connectic applications thanks to its possibility to recover an original shape by heating. Shape memory rings are deformed from a martensitic state, placed around two pipes between which tightness is required, and then heated. Once the shape memory ring recovers its original shape, a contact pressure appears between ring and pipes, and sealing is ensured. Many numerical models $[1,2,3,4]$ permit to model shape memory behavior and design this kind of applications.

However, NiTi transformation hysteresis is relatively small (between 20 and $40{ }^{\circ} \mathrm{C}$ ), and applications are limited into a narrow range of temperature. To enlarge the field of use, $\mathrm{Nb}$ is added to NiTi to obtain $N i T i N b$ ternary shape memory alloy, especially commercial $N i_{47} T i_{44} N b_{9}$ whose transformation temperature hysteresis could be upper than $100{ }^{\circ} \mathrm{C}[5]$. The niobium is present under ductile inclusions with low yield stress into a NiTi shape Memory matrix [6], and inclusions plastic strain induces a widening of the effective Shape Memory Alloy hysteresis.

\footnotetext{
${ }^{a}$ e-mail: boris.piotrowski@esstin.uhp-nancy.fr
} 
The aim is to model the behavior of a NiTiNb Representative Volume Element (RVE) identified by experimental observations, taking into account both phase transformation of the matrix and plastic sliding of the inclusions. A two levels homogenization scheme is also adopted. The first level consists of modeling the SMA matrix behavior from a polycrystalline alloy to an equivalent homogeneous medium (EHM) [2] and the second one is to homogenize the behavior of this EHM containing elastic-plastic spherical inclusions, to conduct via Mori Tanaka scheme [7] to a final EHM. The model is then implemented in a Finite Element code and some applications are simulated. Results are discussed in order to validate the model.

\section{SEM observations}

In order to define the RVE composition, some observations have been made by SEM on $N i_{47} T i_{44} N b_{9}$ sample provided by Intrinsic Devices. Specimens have been cut with low speed diamond saw, then polished with paper containing grains until $0.5 \mu \mathrm{m}$. The micrographies in Figure 1 show the microstructure of the alloy in the two perpendicular plans.

Four phases are observed. The first one is the matrix, which appears in low grey in micrographs, and which corresponds to a NiTi shape memory matrix. The second one is composed of long white ellipsoidal inclusions, which correspond to rich-Niobium phase. The third one is composed of high grey particles. A SEM analysis shows that they correspond to $\mathrm{Ti}_{3}(\mathrm{Ni}, \mathrm{Nb})_{2}$ inclusions, as observed by Zhang et al. [6]. The last one is composed of dark particles, mainly composed of silicon, due to polishing step. Image analysis shows that matrix surface fraction is about $87.5 \%$, rich-Niobium inclusion about $10.7 \%$ and $T i_{3}(N i, N b)_{2}$ inclusions about $1.5 \%$. In order to model the behavior of NiTiNb, a RVE should be established. Neither the silicon particles obtained by polishing error, nor the Ti-rich particles have to be considered. For the last one, it is explained by the poor fraction of inclusion, which does not represent the real behavior. What's more, Ti-rich inclusions are often embedded by Nb-rich phase which is more compliant, so during mechanical loading the $\mathrm{Ti}_{3}(\mathrm{Ni}, \mathrm{Nb})_{2}$ impact is limited, and inclusions tend to act as a rigid body. The RVE has consequently to be composed of NiTi Matrix with ellipsoidal Nb-rich inclusion, as shown in Figure 2.

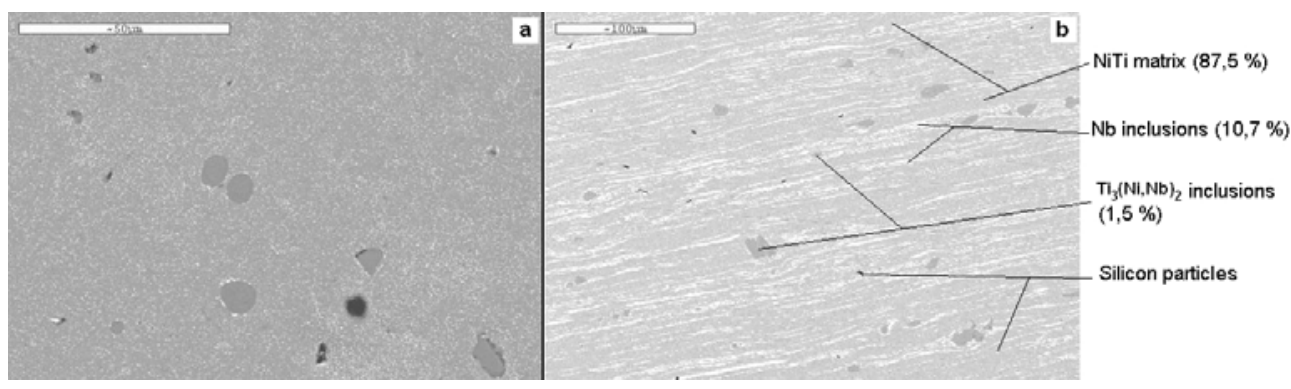

Fig. 1. Micrographies in two plans: a) longitudinal; b) transverse

\section{RVE Modeling}

\subsection{Homogenization strategy}

In order to lead to the thermo mechanical constitutive law, the polycrystalline aggregate of NiTi matrix is described with a macroscopic model developed by Peultier et al. [1], and improved by Chemisky et al. [2]. Inclusions are assumed having an elastic-plastic behavior described by a power consitutive law. Constitutive equations can be written as follows:

$$
\dot{\sigma}_{i j}^{M}(r)=L_{i j k l}^{M}(r) \dot{\varepsilon}_{k l}^{M}(r)-M_{i j}^{M} \dot{T}
$$




$$
\dot{\sigma}_{i j}^{I}(r)=L_{i j k l}^{I}(r) \dot{\varepsilon}_{k l}^{I}(r)-C_{i j k l}^{I}(r) \alpha_{k l}^{I} \dot{T}
$$

$L^{M}$ and $M^{M}$ are respectively the matrix mechanical and thermal tangent operators, and $L^{I}$ and $\alpha^{I}$ are respectively the inclusion mechanical and thermal tangent operators. $C^{I}$ is the inclusion elasticity tensor. Experimental observations show that a matrix phase is clearly identified, and Nb-rich inclusions are in a relatively little fraction (10\%), and well distributed. Consequently, the Mori Tanaka scheme is also adopted and the matrix material is chosen for the reference medium, in order to lead to the effective behavior. Inclusions are assumed to be spherical.

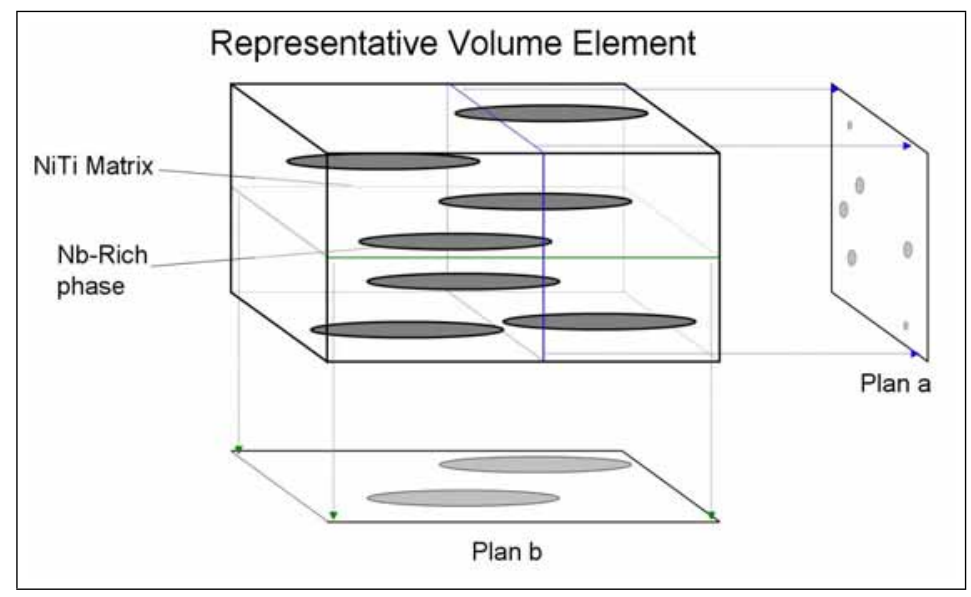

Fig. 2. Representative Volume Element

\subsection{Mori-Tanaka solution}

For modeling, strain rate in boundary of inclusion is assumed equal to the strain rate in matrix $[8]$.

$$
\dot{\varepsilon}_{i j}^{I}=\dot{\varepsilon}_{i j}^{M}+T_{i j k l}^{I I}\left(L_{k l m n}^{M}-L_{k l m n}^{I}\right) \dot{\varepsilon}_{m n}^{I}
$$

where $I$ and $M$ denote respectively Inclusion and Matrix. What's more, the global strain rate is assumed equal to the sum between inclusion strain rate and matrix strain rate, each pondered by its fraction volume.

$$
\dot{E}_{i j}=f \dot{\varepsilon}_{i j}^{I}+(1-f) \dot{\varepsilon}_{i j}^{M}
$$

The concentration fourth order tensor is derived from equations 3 and 4, and strain inside matrix and inclusion can also be determined starting from macroscopic one as follow:

$$
\begin{aligned}
\dot{\varepsilon}_{m n}^{I} & =A_{m n i j}^{M T} \dot{E}_{i j} \\
\dot{\varepsilon}_{m n}^{M} & =\frac{1}{1-f}\left(\left(I_{m n i j}-f A_{m n i j}^{M T}\right) \dot{E}_{i j}\right)
\end{aligned}
$$

with $A_{m n i j}^{M T}=\left[I_{i j m n}-(1-f) T_{i j k l}^{I I}\left(L_{k l m n}^{M}-L_{k l m n}^{I}\right)\right]^{-1}$. Strain in inclusion and in matrix can derived from macroscopic strain (5). Consequently, by taking into account the constitutive equations for matrix (1) and inclusion (2), the effective constitutive law is derived as follow:

$$
\dot{\Sigma}_{i j}=f \dot{\sigma}_{i j}^{I}+(1-f) \dot{\sigma}_{i j}^{M}=L_{i j m n}^{e f f} \dot{E}_{m n}-M_{i j}^{e f f} \dot{T}
$$

with $L_{i j m n}^{e f f}=f\left(L_{i j k l}^{I}-L_{i j k l}^{M}\right) A_{k l m n}^{M T}+L_{i j m n}^{M}$ and $M_{i j}^{e f f}=f C_{i j k l}^{I} \alpha_{k l}^{I}+(1-f) M_{i j}^{M}$. 
Tab. 1. SMA Matrix parameter values

\begin{tabular}{lllllll}
\hline$E^{M}(\mathrm{MPa})$ & $\nu^{M}$ & $\alpha^{M}$ & $\left(\varepsilon_{\text {trac }}^{T}\right)^{\max }$ & $\left(\varepsilon_{\text {comp }}^{T}\right)^{\max }$ & $\mathrm{b}\left(\mathrm{MPa} /{ }^{\circ} \mathrm{C}\right)$ & $\mathrm{Ms}\left({ }^{\circ} \mathrm{C}\right)$ \\
\hline 70000 & 0.3 & 0 & 0.05 & 0.04 & 5 & -57 \\
\hline Af $\left({ }^{\circ} \mathrm{C}\right)$ & $r_{f}$ & $F_{\varepsilon}(\mathrm{MPa})$ & $H_{f}(\mathrm{MPa})$ & $H_{\varepsilon}(\mathrm{MPa})$ & $H_{\text {twin }}(\mathrm{MPa})$ & \\
\hline 5 & 0.7 & 160 & 5 & 2100 & 40000 & \\
\hline
\end{tabular}

Tab. 2. Elastic-plastic parameter values

\begin{tabular}{lllllll}
\hline & $E^{I}(\mathrm{MPa})$ & $\nu^{I}$ & $\alpha^{I}$ & $\sigma^{Y}(\mathrm{MPa})$ & $H^{\text {iso }}(\mathrm{MPa})$ & $\mathrm{n}$ \\
\hline case 1 & 53000 & 0.3 & 0 & 90 & 700 & 4 \\
case 2 & 53000 & 0.3 & 0 & 700 & 70 & 4 \\
\hline
\end{tabular}

\section{Results and discussion}

Numerical simulations, for various loadings, based on the developed model are presented. The model has been implemented in the commercial Finite Element Code Abaqus, via the User MATerial UMAT subroutine.

\subsection{Inclusion parameters influence on As temperature}

Some simulations have been conducted on only one integration point in order to hightlight inclusion impact on effective thermomechanical behavior. The point, initialy into austenitic state, is cooled from $100{ }^{\circ} \mathrm{C}$ to $-100{ }^{\circ} \mathrm{C}$ and martensite is formed. A pressure of $900 \mathrm{MPa}$ is applied, and is then relaxed. During this step, which can be identified as a SMA component education, both martensitic orientation and plastic sliding are activated, respectively in matrix and inclusions. After having locked the strain, the element is finally heated, in order to activate the reverse transformation and induce stress increasing. Two cases are considered, the differences between them are the inclusion yield stress and the isotropic hardening parameter values. The shape memory matrix material parameter values are given in Table 1 and elastic-plastic material parameter are given in Table 2 .

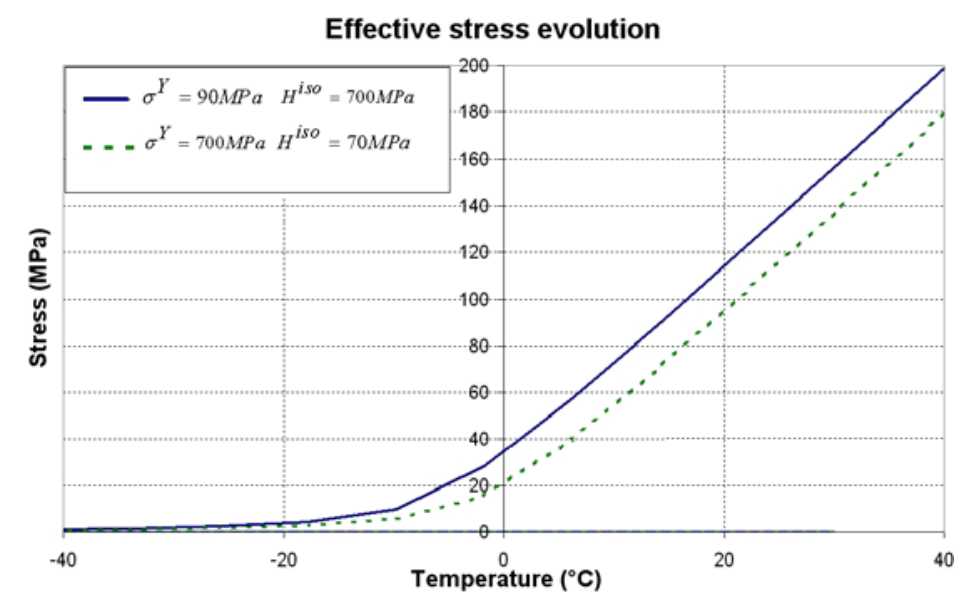

Fig. 3. As increasing after predeformation 
The Figure 3 shows that the model takes into account the predeformation impact on As reverse transformation temperature, and the properties of the inclusion on the increasing level. Indeed, the plastic strain due to predeformation in the inclusions modifies the reverse transformation temperature. However, the As increasing is upper of $5{ }^{\circ} \mathrm{C}$ in the second case compared with first case, whereas inclusion are less ductile: the influence is not directly due to the ability of inclusion to have plastic strain. The As increasing is due to the stress value in matrix and inclusion, after predeformation. The Figure 4 shows the stress evolution with strain into each phase, in the two considered cases.
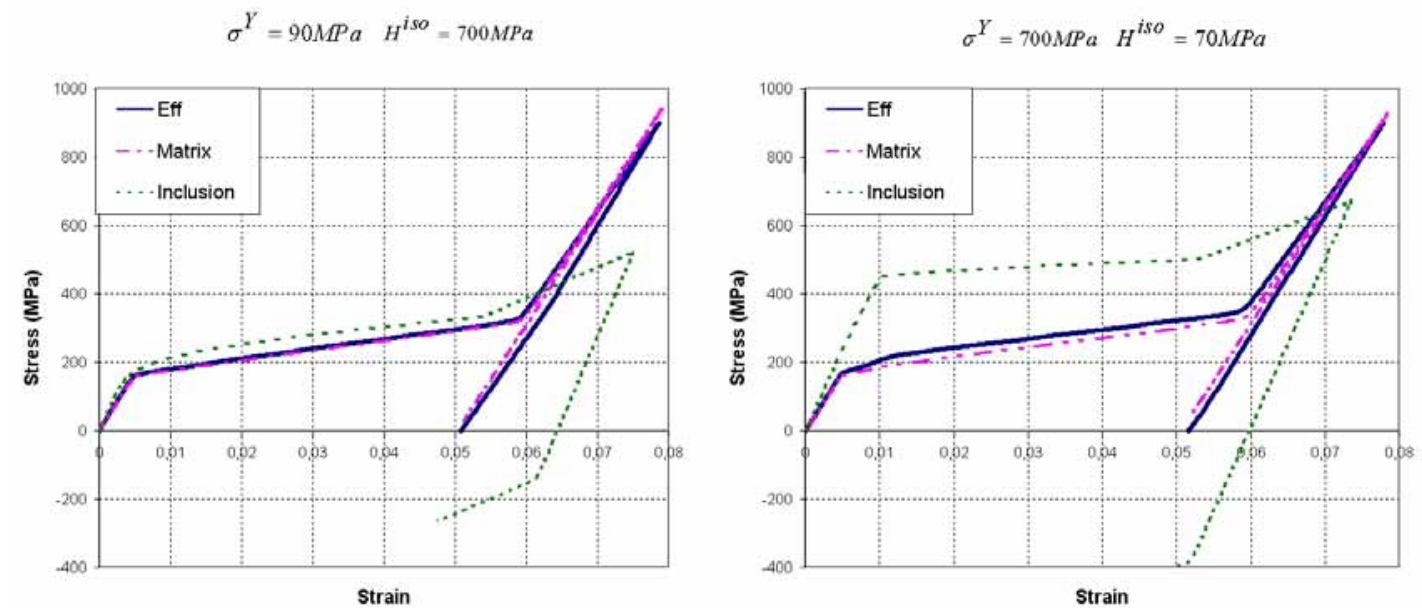

Fig. 4. Stress evoltution during predeformation in each case

To explain the increasing process, we have to focus on the SMA matrix behavior. In the first case, the residual stress in the matrix after predeformation is about $42 \mathrm{MPa}$, whereas it is about $67 \mathrm{MPa}$ in the second case. During the heating, the internal stress will delay the beginning of the reverse transformation, as illustrated on Figure 5.

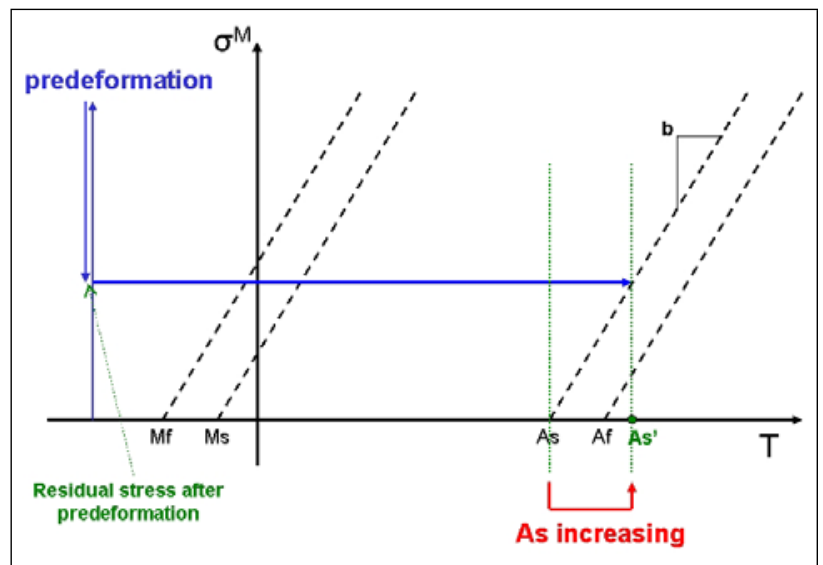

Fig. 5. As temperature increasing explanation 
The difference of $25 \mathrm{MPa}$ induces an increasing of As about $5{ }^{\circ} \mathrm{C}$, because $b$ material parameter which describe the transformation temperatures evolution with stress level is equal to $5 \mathrm{MPa} /{ }^{\circ} \mathrm{C}$. This result, only qualitative, validates that the model is able to predict the reverse transformation temperature evolution. However, the SMA matrix parameters have also an important influence on the value of increasing, and these parameters are being studied. What's more, He and Rong have experimentally shown that the predeformation influence on As temperature has a non linear evolution, and became upper than $50{ }^{\circ} \mathrm{C}$ from a predeformation level of $8 \%$ [5]. At this level, the stress in the SMA matrix is upper than plastic yield stress, and the plastic strain of the matrix should modify the value of internal remaining stress in SMA. Plastic behavior of shape memory matrix has to be considered in order to simulate this predeformation above $8 \%$, further investigations are in progress.

\subsection{Ring application: comparison between $\mathrm{NiTi}$ and $\mathrm{NiTiNb}$}

Three simulations have been made on the same device, but considering three different materials. The device consists of a martensitic SMA ring which is opened at $-100{ }^{\circ} \mathrm{C}$, placed around a pipe and heated until $100{ }^{\circ} \mathrm{C}$ in order to have a contact pressure. The results of NiTi and predeformed NiTiNb simulations are shown in Figure 7. The material parameters chosen have to describe NiTi, NiTiNb and predeformed NiTiNb resumed in Table 3 and 4 respectively. In NiTi case, the constitutive law describes a shape memory behaviour without inclusion, with transformation temperatures around room temperature. In NiTiNb case, Ms temperature value is $-55{ }^{\circ} \mathrm{C}$, and As temperature depends on predeformation level. DSC analyses have been performed on NiTiNb studied, and results show that As temperature is about $5{ }^{\circ} \mathrm{C}$ without predeformation, and about $63{ }^{\circ} \mathrm{C}$ after a predeformation estimated to $14 \%$ [5].

Tab. 3. NiTi material parameters

\begin{tabular}{lllllll}
\hline$E^{M}(\mathrm{MPa})$ & $\nu^{M}$ & $\alpha^{M}$ & $\left(\varepsilon_{\text {trac }}^{T}\right)^{\max }$ & $\left(\varepsilon_{\text {comp }}^{T}\right)^{\max }$ & $\mathrm{b}\left(\mathrm{MPa} /{ }^{\circ} \mathrm{C}\right)$ & $\mathrm{Ms}\left({ }^{\circ} \mathrm{C}\right)$ \\
\hline 70000 & 0.3 & 0 & 0.05 & 0.04 & 5 & 10 \\
\hline Af $\left({ }^{\circ} \mathrm{C}\right)$ & $r_{f}$ & $F_{\varepsilon}(\mathrm{MPa})$ & $H_{f}(\mathrm{MPa})$ & $H_{\varepsilon}(\mathrm{MPa})$ & $H_{t w i n}(\mathrm{MPa})$ & \\
\hline 35 & 0.7 & 160 & 5 & 2000 & 40000 & \\
\hline
\end{tabular}

Tab. 4. NiTiNb material parameters

\begin{tabular}{lllllll}
\hline$E^{M}(\mathrm{MPa})$ & $\nu^{M}$ & $\alpha^{M}$ & $\left(\varepsilon_{\text {trac }}^{T}\right)^{\max }$ & $\left(\varepsilon_{\text {comp }}^{T}\right)^{\max }$ & $\mathrm{b}\left(\mathrm{MPa} /{ }^{\circ} \mathrm{C}\right)$ & $\mathrm{Ms}\left({ }^{\circ} \mathrm{C}\right)$ \\
\hline 70000 & 0.3 & 0 & 0.05 & 0.04 & 5 & -57 \\
\hline Af $\left({ }^{\circ} \mathrm{C}\right)$ & $r_{f}$ & $F_{\varepsilon}(\mathrm{MPa})$ & $H_{f}(\mathrm{MPa})$ & $H_{\varepsilon}(\mathrm{MPa})$ & $H_{t w i n}(\mathrm{MPa})$ & \\
\hline $5 / 63$ & 0.7 & 160 & 5 & 4000 & 40000 & \\
\hline$E^{I}(\mathrm{MPa})$ & $\nu^{I}$ & $\alpha^{I}$ & $\sigma^{Y}(\mathrm{MPa})$ & $H^{i s o}(\mathrm{MPa})$ & $\mathrm{n}$ & \\
\hline 53000 & 0.3 & 0 & 90 & 700 & 4 & \\
\hline
\end{tabular}

The Figure 6 shows the contact pressure evolution during heating and cooling steps. 


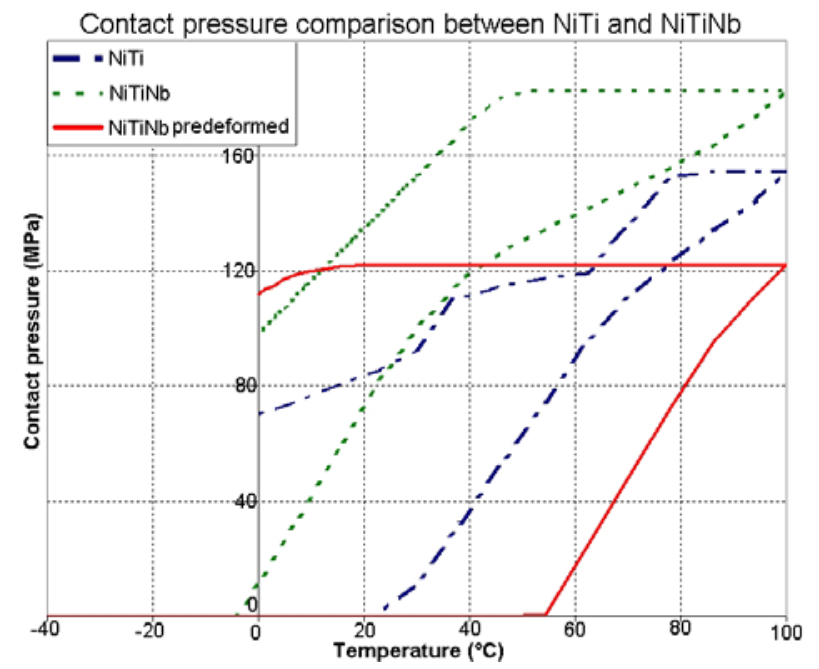

Fig. 6. Contact pressure evolution

The first point is that NiTiNb alloys permit to minimize the contact pressure descreasing when the assembly returns to room temperature. Indeed, the low martensitic transformation temperatures of NiTiNb, compared with NiTi, permit to delay the return to martensitic phase during cooling. This is the main advantage of NiTiNb alloys.

Nevertheless, the contact pressure with NiTi is upper than NiTiNb case. This is explained by the fact that the control variable in this part of simulation is the temperature, and in each case the heating has been applied until the same tempature, $100{ }^{\circ} \mathrm{C}$. It should be noted that martensite fraction in NiTi is greater than in NiTiNb case at the same temperature. This means that the maximal contact pressure does not appear at the same temperature in NiTi and NiTiNb cases, but at a higher one in the second case.

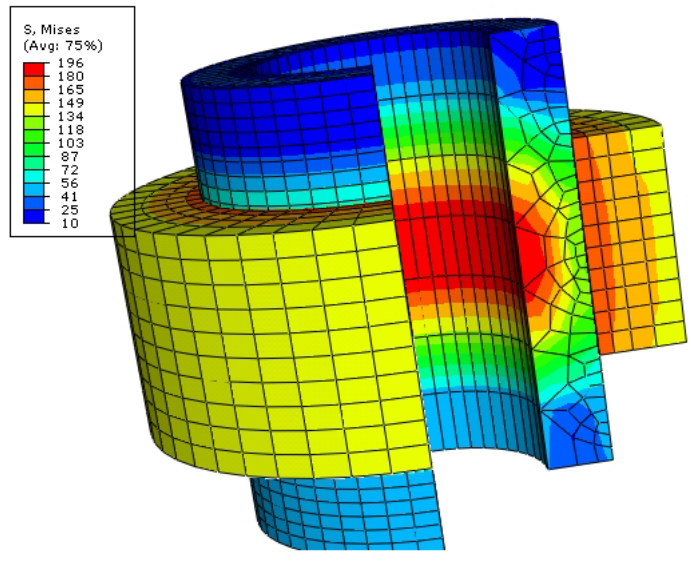

NiTi case

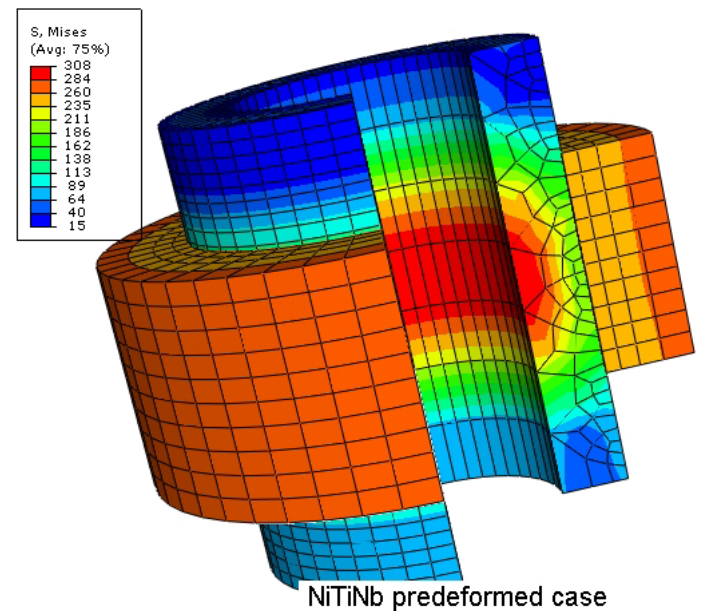

NiTiNb predeformed case

Fig. 7. Stress (MPa) in NiTi and NiTiNb predeformed Ring at the end of applied cycle 


\section{Conclusion}

A two phases model is proposed which permits to take into account Niobium inclusions in NiTiNb shape memory alloys. Experimental observations induce that the Mori-Tanaka homogenization scheme is adopted, and an effective behavior law is formulated. The implementation in a Finite Element code and simulations have validated the qualitative behavior of the studied alloy. However, some parametric studies have to be conducted in order to fit the NiTiNb material parameters, because they can not all be identify directly by experimental investigations. What's more, we have seen that the predeformation simulation needs to take into account the plastic sliding into the shape memory matrix, investigations are under development.

\section{Références}

1. B. Peultier, T. Ben Zineb, E. Patoor, Materials Science and Engineering A 481-482, (2008) 383-388

2. Y. Chemisky, A. Duval, B. Piotrowski, T. Ben Zineb, V. Tahiri, E. Patoor, Smart Materials and Structures, (2009) accepted

3. M. Panico, L. C. Brinson, Journal of the Mechanics and Physics of Solids 55, (2007) 2491-2511

4. P. Popov, D. C. Lagoudas, International Journal of Plasticity 23, (2007) 1679-1720

5. X. M. He, L. J. Rong, Scripta Materialia 51, (2004) 7-11

6. C. S. Zhang, Y. Q. Wang, W Chai, L. C. Zhao, Materials Chemistry and Physics 28, (1991) 43-50

7. T. Mori, K. Tanaka, Acta Metallurgica 21, (1973) 571-574

8. J. D. Eshelby, Progress in Solid Mechanics 2, (1961) 89 